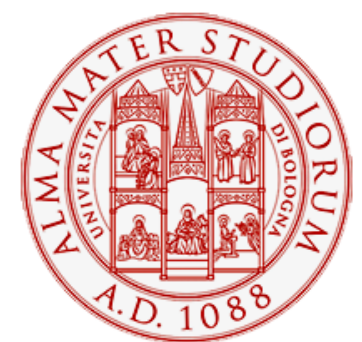

Alma Mater Studiorum - Università di Bologna DEPARTMENT OF ECONOMICS

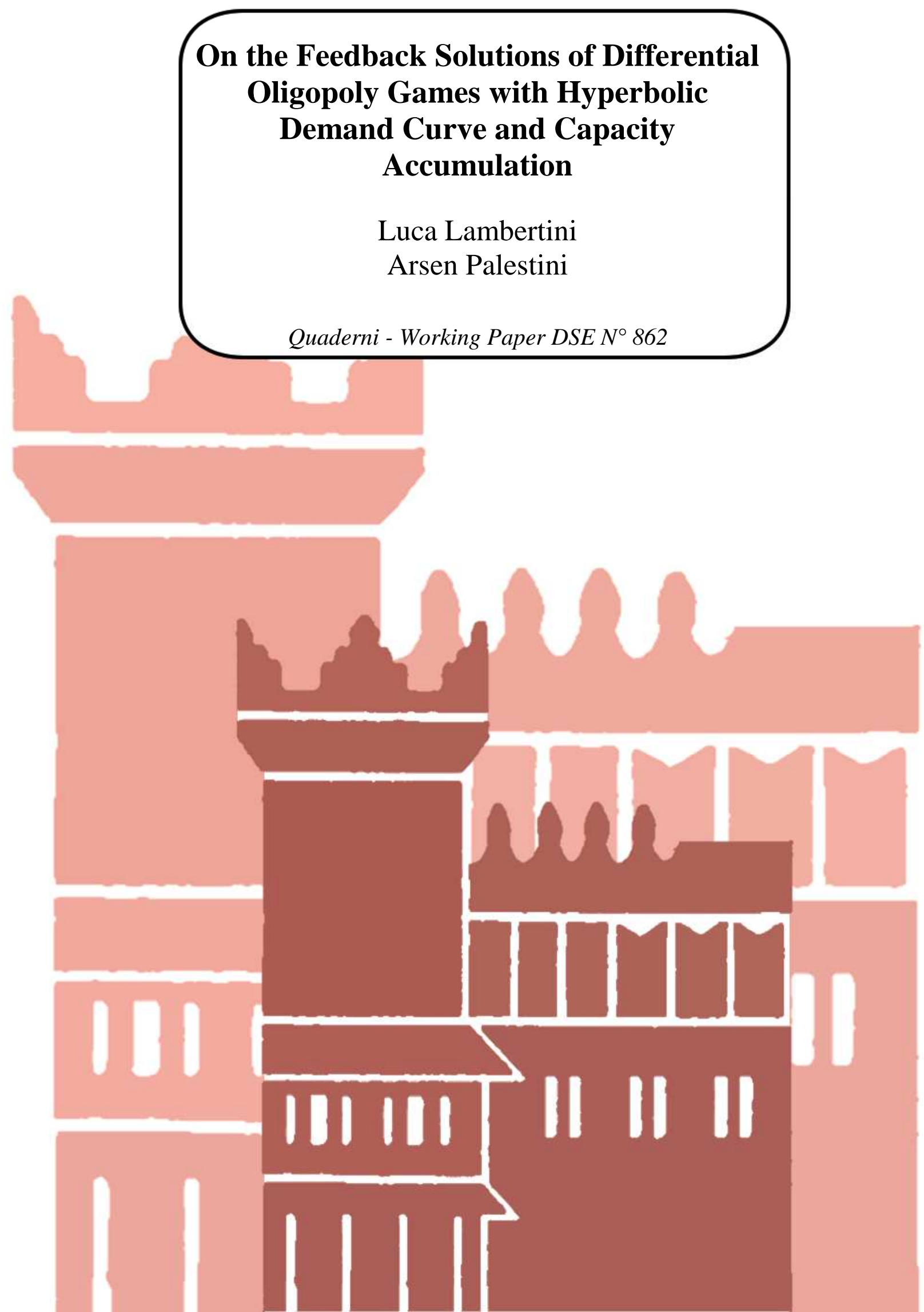




\title{
On the Feedback Solutions of Differential Oligopoly Games with Hyperbolic Demand Curve and Capacity Accumulation ${ }^{1}$
}

\author{
Luca Lambertini ${ }^{*}$, Arsen Palestini ${ }^{\sharp}$ \\ *Department of Economics, University of Bologna \\ Strada Maggiore 45, 40125 Bologna, Italy; fax +39-051-2092664 \\ luca.lambertini@unibo.it \\ $\S$ ENCORE, University of Amsterdam \\ Roeterstraat 11, WB1018 Amsterdam, The Netherlands \\ $\sharp$ MEMOTEF, Sapienza University of Rome
}

Via del Castro Laurenziano 9, 00161 Rome, Italy; arsen.palestini@uniroma1.it

9th January 2013

\footnotetext{
${ }^{1}$ We would like to thank Davide Dragone and Alessandro Tampieri for stimulating discussions and insightful suggestions. The usual disclaimer applies.
} 


\begin{abstract}
We characterise the subgame perfect equilibrium of a differential market game with hyperbolic inverse demand where firms are quantity-setters and accumulate capacity over time à la Ramsey. The related Hamilton-JacobiBellman are solved in closed form both on infinite and on finite horizon setups and the optimal strategies are determined. Then, we analyse the feasibility of horizontal mergers in both static and dynamic settings, and find appropriate conditions for their profitability under both circumstances. Static profitability of a merger implies dynamic profitability of the same merger. It appears that such a demand structure makes mergers more likely to occur than they would on the basis of the standard linear inverse demand.
\end{abstract}

JEL Classification: C73, L13

Keywords: capacity, differential game, Markov-perfect equilibrium, Hamilton-Jacobi-Bellman equation, horizontal mergers 


\section{Introduction}

Most of the existing literature on oligopoly theory (either static or dynamic) assumes linear inverse demand functions, as this, in addition to simplifying calculations, also ensures both concavity and uniqueness of the equilibrium, which, in general, wouldn't be warranted in presence of convex demand systems (see [14] and [8], inter alia). However, the use of linear demand function is in sharp contrast with the standard microeconomic approach to consumer behaviour, where the widespread adoption of Cobb-Douglas preferences (or their log-linear affine transformation) yields hyperbolic inverse demand functions. The same applies to the so-called quasi-linear utility function, concave in consumption and linear in money, that again yields a convex demand system. Indeed, both preference structures share the common property of producing isoelastic demand functions. ${ }^{1}$

In fact, this is sometimes openly referred to in the field of industrial organization, where researchers mention the opportunity of dealing with non-linear demand curves, and then promptly leave it aside for the sake of tractability. ${ }^{2}$ Additionally, the econometric approach to demand theory has produced the highest efforts to building up a robust approach to the estimation of non-linear individual and market inverse demand functions, yielding a large empirical evidence in this direction. ${ }^{3}$ With these considerations in mind, it appears desirable to investigate the bearings of non-linear demand systems on the performance of firms operating in oligopolistic markets, using thus a setup with solid microfoundations corroborated by robust empirical evidence, even though this is a costly approach in terms of analytical tractability.

\footnotetext{
${ }^{1}$ For a thorough illustration of these issues in consumer theory, see the classical textbooks: [7] and [27], inter alia.

${ }^{2} \mathrm{~A}$ noteworthy example in this respect is [23] (pp. 53-54), using quasi-linear utility function to define the concept of consumer surplus.

${ }^{3}$ See [15], [25] and [26], inter alia.
} 

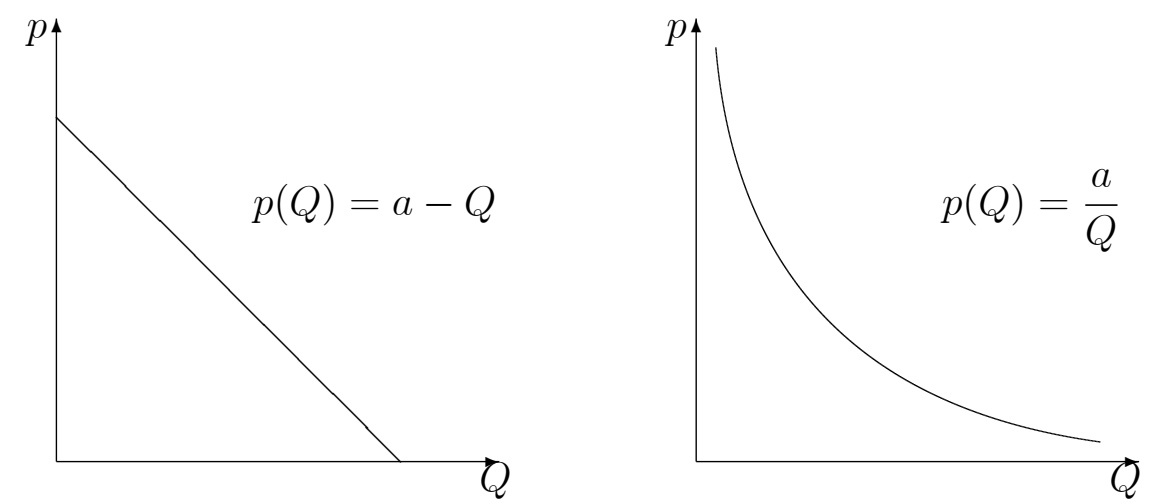

Figure 1. Inverse demand functions in the linear and in the hyperbolic cases

With specific reference to differential games, the use of linear inverse demand functions (jointly with either linear or quadratic cost functions) allows for the closed-form solution of the feedback equilibrium through the Bellman equation of the representative firm, as the model takes a linearquadratic form and therefore one can stipulate that the corresponding candidate value function is also linear-quadratic. However, there is no particular reason to believe that a linear function describes correctly virtually any market demand in the real world, and therefore it is of primary interest to design, if possible, closed-form solutions of market games with non-linear demand functions. To the best of our knowledge, the only existing examples of differential oligopoly games with non-linear market demand are in [5], [11] and [18]. The first one uses a non-linear demand à la Anderson and Engers ([1]) and also investigates horizontal mergers, whereas the second one carries out a profitability assessment of small horizontal mergers subject to a sticky price dynamics. The third one employs a hyperbolic demand with sticky prices (as in [24] and [13]) as well, but leaves the merger issue out of the picture. Other non linear-quadratic structures are investigated by [16] and $[17] .^{4}$

The aim of this paper is to illustrate a way out of the aforementioned problem, offered by dynamic game theory. We are going to illustrate a dynamic Cournot model where firms (i) accumulate capacity à la Ramsey (1928), (ii) bear an instantaneous cost of holding any given capacity, and (iii) discount future profits at a constant rate. The main results are threefold.

\footnotetext{
${ }^{4}$ For excellent and largely complementary surveys of differential oligopoly games, see [9] and [19].
} 
1. First, we determine the feedback information structure of the differential game both over infinite and finite horizon, solving in closed form the related Hamilton-Jacobi-Bellman systems of equations when the accumulation dynamics is linear and the accumulation cost is a polynomial function of a generic degree of the firm's capital endowment. When the accumulation follows a linear growth dynamics, the resulting feedback equilibrium coincides with the open-loop equilibrium, hence it is indeed subgame perfect.

2. Secondly, we investigate the standard Ramsey $A k$ model where the inverse demand is a hyperbolic curve. By applying the above results, we are able to completely characterize the equilibrium structure. Some results on feasibility of states and controls and on the comparison between the firms' profits in the static and in the dynamic frameworks are also featured.

3. Finally, we use it to investigate the profit (or, private) incentive towards horizontal mergers, to find that taking a dynamic perspective widens the range of privately feasible mergers. In particular, the form of the demand curve is crucial to allow for profitable mergers to take place in all setups: $:^{5}$ That is, the presence of discounting, depreciation and a cost associated to holding capacity increases the firms' willingness to merge horizontally, for any admissible merger size. Any merger, of course, has undesirable consequences on consumer surplus and ultimately for welfare (at least in this model, where the efficiency defense is not operating).

The remainder of the paper is structured as follows. Section 2 features the basic concepts on the static game, and subsequently the complete calculation and the related properties of the feedback information structure of the differential game with capacity accumulation over infinite and finite horizons. In Section 3 two applications are taken into account and characterized: the Cournot-Ramsey differential game and the analysis of profitability of horizontal mergers. Section 4 incorporates our conclusions and further possible developments.

\footnotetext{
${ }^{5}$ To the best of our knowledge, scant attention has been devoted to the implications of dynamic competition on merger incentives, with the exceptions of Dockner and Gaunersdorfer in 2001 ([9]) and Benchekroun in 2003 ([2]), using a price dynamics à la Simaan and Takayama ([24]) and Cellini and Lambertini ([5]) adopting a Ramseytype capital accumulation dynamics. All of these contributions, however, assume linear demand functions.
} 


\section{The oligopoly game with hyperbolic in- verse demand}

\subsection{A summary of the static game}

Consider a market where $N$ single-product firms supply individual quantities $q_{i}>0, i=1, \ldots, N$. The good is homogeneous, and the market inverse demand function is

$$
p\left(q_{1}, \ldots, q_{N}\right)=\frac{a}{q_{1}+\cdots+q_{N}}=\frac{a}{Q} .
$$

(1) is the outcome of the constrained maximum problem of a representative consumer endowed with a log-linear utility function

$$
U(Q)=\log [Q]+m,
$$

where $m$ is a numeraire good whose price is normalised to one. The budget constraint establishes that the consumer's nominal income $Y$ must be large enough to cover the expenditure, so that $Y \geq p(Q) Q+m$. The representative consumer is supposed to solve the following:

$$
\max _{Q} L(Q)=U(Q)+\mu(Y-p(Q) Q-m) .
$$

Solving the above problem, one obtains indeed the hyperbolic inverse demand function (1), where $a=\mu^{-1}>0$.

On the supply side, production entails a total cost $C_{i}=c q_{i}$, where $c>0$ is a constant parameter measuring marginal production cost. Market competition takes place à la Cournot-Nash; therefore, firm $i$ chooses $q_{i}$ so as to maximise profits $\pi_{i}=(p(Q)-c) q_{i}$. This entails that the following first order conditions must be satisfied (given their form, it is not necessary to assume interior solutions):

$$
\frac{\partial \pi_{i}}{\partial q_{i}}=\frac{a Q_{-i}}{\left(q_{i}+Q_{-i}\right)^{2}}-c=0
$$

where $Q_{-i} \equiv \sum_{j \neq i} q_{j}$. The associated second order condition:

$$
\frac{\partial^{2} \pi_{i}}{\partial q_{i}^{2}}=-\frac{2 a Q_{-i}}{\left(q_{i}+Q_{-i}\right)^{3}}<0
$$

is always met when $q_{i}>0$ for all $i=1, \ldots, N$. Then, imposing the symmetry condition on all outputs, i.e. $q_{i}=q_{j}=q$, one obtains the 
individual Cournot-Nash equilibrium output $q^{C N}=\frac{a(N-1)}{N^{2} c}$, yielding profits $\pi^{C N}=\frac{a}{N^{2}}$. If the $N$ firms were operating under perfect competition, then $p^{*}=c$ and therefore $q^{*}=\frac{a}{N c}$.

It is apparent that the above solutions (i.e., both the Cournot-Nash equilibrium and the perfectly competitive equilibrium) are well-defined and feasible for all $c>0$.

In the remainder of the paper, we will turn our attention to a differential game where the demand structure is the same as here. We will separately investigate the infinite horizon and finite horizon cases.

\subsection{Feedback solutions of the differential game on an infinite horizon}

We are going to consider a market existing over $t \in[0,+\infty)$, and which is served by $N$ firms producing a homogeneous good. Let $q_{i}(t) \in(0,+\infty)$ define the quantity sold by firm $i$ at time $t$. Firms compete à la Cournot, the demand function at time $t$ being:

$$
p(t)=\frac{a}{Q(t)}, \quad Q(t)=\sum_{i=1}^{N} q_{i}(t) .
$$

In order to produce, firms bear linear instantaneous costs $C_{i}(t)=c q_{i}(t)$, where $C>0$. Moreover, they must accumulate capacity or physical capital $k_{i}(t) \in[0,+\infty)$ over time. If we denote with $y_{i}(t)$ the output produced by firm $i$ at time $t$, we assume that $k_{i}$ affects the production of $y_{i}$ in the sense that $\frac{\partial y_{i}}{\partial k_{i}}>0$. Capital accumulates as a result of intertemporal relocation of unsold output $y_{i}(t)-q_{i}(t) .{ }^{6}$ This can be interpreted in two ways. The first consists in viewing this setup as a corn-corn model, where unsold output is reintroduced in the production process. The second consists in thinking of a two-sector economy where there exists an industry producing the capital input which can be traded against the final good at a price equal to one (for further discussion, see [5]). Unlike the standard macroeconomic approach to growth models in a Ramsey fashion, here we will allow for the presence of an instantaneous cost of holding installed capacity. This cost will be $\Gamma_{i}\left(k_{i}(t)\right)$, possibly asymmetric across firms. In the remainder, we will refer to $\frac{\partial \Gamma_{i}}{\partial k_{i}}$ as a measure of the opportunity cost of a unit of capacity. We will

\footnotetext{
${ }^{6}$ Of course, capacity decumulates whenever $y_{i}(t)-q_{i}(t) \leq 0$.
} 
employ $k_{i}(t)$ as the $i$-th state variable subject to the following dynamic constraints:

$$
\left\{\begin{array}{l}
\frac{d k_{i}(t)}{d t} \equiv \dot{k}_{i}(t)=G_{i}\left(k_{i}(t)\right)-q_{i}(t) \\
k_{i}\left(t_{0}\right)=k_{i 0}>0
\end{array},\right.
$$

where $G_{i}\left(k_{i}(t)\right)$ is a $C^{2}\left(\mathbb{R}_{+}\right)$function affecting the growth dynamics of capital. The $i$-th firm's strategic variable is $q_{i}(t)$, while the $i$-th firm's state variable is $k_{i}(t)$.

Assuming all firms discount profits at the same constant rate $\rho \geq 0$, the problem of firm $i$ is to choose the output level $q_{i}(t)$ so as to maximise its own discounted flow of profits (from now on, we will omit time arguments whenever possible):

$$
\begin{gathered}
\max _{q_{i} \in \mathbb{R}_{+}} J_{i}\left(k_{i 0}, t_{0}\right) \equiv \int_{t_{0}}^{\infty}\left[(p(Q(\tau), \tau)-c) q_{i}(\tau)-\Gamma_{i}\left(k_{i}(\tau)\right)\right] e^{-\rho\left(t_{0}-\tau\right)} d \tau= \\
=\int_{t_{0}}^{\infty}\left[\left(\frac{a}{\sum_{i=1}^{N} q_{i}(\tau)}-c\right) q_{i}(\tau)-\Gamma_{i}\left(k_{i}(\tau)\right)\right] e^{-\rho\left(t_{0}-\tau\right)} d \tau
\end{gathered}
$$

Call $k_{i 0}=k$. Denoting with $V_{i}(k)$ the $i$-th optimal value function for (8), the Hamilton-Jacobi-Bellman (HJB) system of equations reads as follows:

$$
\rho V_{i}(k)=\max _{q_{i}}\left\{\left(\frac{a}{\sum_{i=1}^{N} q_{i}}-c\right) q_{i}-\Gamma_{i}(k)+\frac{\partial V_{i}}{\partial k}\left(G_{i}(k)-q_{i}\right)\right\},
$$

for all $i=1, \ldots, N$. Note that because $J_{i}$ only depends on the $i$-th capital, in (9) the first order partial derivatives of $V_{i}$ with respect to the remaining state variables do not appear. To proceed with the analytical solution of the feedback problem, we are going to introduce suitable symmetry conditions: one is $q_{i}=q_{j}$ for all $i \neq j$, saying that the equilibrium output must be symmetric across all firms. The assumption of symmetry across capitals states that, from the standpoint of a generic firm $i$, the rivals' capacities (and therefore also their weights in the value function) must be symmetric when the respective growth dynamics and cost structures are equal.

Proposition 1. Assuming symmetry across all variables, the HJB equation of the problem is given by:

$$
\frac{\partial V}{\partial k}=\rho \frac{V(k)}{G(k)}+\frac{\Gamma(k)-\frac{a}{N^{2}}}{G(k)},
$$


Proof. Maximizing the r.h.s. of (9) with respect to $q_{i}$ yields:

$$
\frac{a Q_{-i}}{\left(q_{i}+Q_{-i}\right)^{2}}-c-\frac{\partial V_{i}}{\partial k}=0
$$

then, by assuming symmetry on the relevant variables and functions, i.e. $q_{1}=\ldots=q_{N}=q, V_{1}=\ldots=V_{N}=V, \Gamma_{1}(\cdot)=\ldots=\Gamma_{N}(\cdot)=\Gamma(\cdot), G_{1}(\cdot)=$ $\ldots=G_{N}(\cdot)=G(\cdot)$, we have that (11) yields the following expression for the optimal strategy $q^{*}$ :

$$
q^{*}=\frac{a(N-1)}{N^{2}\left(\frac{\partial V}{\partial k}+c\right)}
$$

which must be plugged into (9) to achieve:

$$
\begin{gathered}
\rho V(k)=\left(\frac{N\left(\frac{\partial V}{\partial k}+c\right)}{N-1}-c\right) \frac{a(N-1)}{N^{2}\left(\frac{\partial V}{\partial k}+c\right)}-\Gamma(k)+\frac{\partial V}{\partial k}\left(G(k)-\frac{a(N-1)}{N^{2}\left(\frac{\partial V}{\partial k}+c\right)}\right) \Longleftrightarrow \\
\Longleftrightarrow \rho V(k)=\frac{a\left(N \frac{\partial V}{\partial k}+c\right)}{N^{2}\left(\frac{\partial V}{\partial k}+c\right)}-\Gamma(k)+\frac{\partial V}{\partial k} G(k)-\frac{\partial V}{\partial k}\left(\frac{a(N-1)}{N^{2}\left(\frac{\partial V}{\partial k}+c\right)}\right) \Longleftrightarrow \\
\Longleftrightarrow \rho V(k)+\Gamma(k)-\frac{\partial V}{\partial k} G(k)=\frac{a}{N^{2}\left(\frac{\partial V}{\partial k}+c\right)}\left(N \frac{\partial V}{\partial k}+c-(N-1) \frac{\partial V}{\partial k}\right) \Longleftrightarrow \\
\Longleftrightarrow \rho V(k)+\Gamma(k)-\frac{\partial V}{\partial k} G(k)=\frac{a}{N^{2}\left(\frac{\partial V}{\partial k}+c\right)}\left(\frac{\partial V}{\partial k}+c\right) \Longleftrightarrow \\
\Longleftrightarrow \frac{\partial V}{\partial k}=\rho \frac{V(k)}{G(k)}+\frac{\Gamma(k)-\frac{a}{N^{2}}}{G(k)} .
\end{gathered}
$$

Corollary 2. (10) admits the following family of solutions in any interval properly contained in the set $\left\{k \in \mathbb{R}_{+} \mid G(k) \neq 0\right\}$ :

$$
V^{*}(k)=\left(\widetilde{C}+\int^{k}\left(\frac{\Gamma(s)-\frac{a}{N^{2}}}{G(s)}\right) e^{-\int^{s} \frac{\rho}{G(\tau)} d \tau} d s\right) e^{\int^{k} \frac{\rho}{G(s)} d s},
$$

where $\widetilde{C}$ is a constant depending on the initial conditions of (10). 
Expression (13) is useful to characterize the standard cases. In particular, when the capital's production function is linear and it does not involve fixed costs in absence of capital, it suggests us the following result:

Proposition 3. If $G(k)$ is linear, $G(0)=0$ and $\Gamma(k)$ is an $m$-th degree polynomial in $k$, then one solution of $(10)$ is an $m$-th degree polynomial in $k$ as well.

Proof. By assumption, call $G(k)=\alpha k$ and $\Gamma(k)=\sum_{l=0}^{m} \beta_{l} k^{l}$. Replacing such functions in (13) yields:

$$
\begin{gathered}
V^{*}(k)=\left(\widetilde{C}+\int^{k}\left(\frac{\sum_{l=0}^{m} \beta_{l} s^{l}-\frac{a}{N^{2}}}{\alpha s}\right) e^{-\int^{s} \frac{\rho}{\alpha \tau} d \tau} d s\right) e^{f^{k} \frac{\rho}{\alpha s} d s}= \\
=\left(\widetilde{C}+\int^{k}\left(\frac{\sum_{l=0}^{m} \beta_{l} s^{l}-\frac{a}{N^{2}}}{\alpha s}\right) s^{-\frac{\rho}{\alpha}} d s\right) k^{\frac{\rho}{\alpha}}= \\
=\widetilde{C} k^{\frac{\rho}{\alpha}}+\left[\int^{k}\left(\frac{1}{\alpha} \sum_{l=0}^{m} \beta_{l} s^{l-\frac{\rho}{\alpha}-1}-\frac{a}{\alpha N^{2}} s^{-\frac{\rho}{\alpha}-1}\right) d s\right] k^{\frac{\rho}{\alpha}}= \\
=\widetilde{C} k^{\frac{\rho}{\alpha}}+\sum_{l=0}^{m} \frac{\beta_{l}}{\alpha l-\rho} k^{l}+\frac{a}{\rho N^{2}},
\end{gathered}
$$

hence the solution corresponding to the choice $\widetilde{C}=0$ is an $m$-th degree polynomial in $k$.

By plugging the solution into (12), it follows that:

Corollary 4. If the assumptions of Proposition 3 hold, the optimal feedback strategy is given by:

$$
q^{*}(k)=\frac{a(N-1)}{N^{2}\left(\sum_{l=1}^{m} \frac{l \beta_{l}}{\alpha l-\rho} k^{l-1}+c\right)} .
$$

\subsection{Feedback solutions of the differential game on a finite horizon}

On a finite horizon $[t, T]$, where $0 \leq t<T<\infty$, the HJB system of equations of our problem takes the following form:

$$
-\frac{\partial V_{i}}{\partial t}+\rho V_{i}(k)=\max _{q_{i}}\left\{\left(\frac{a}{\sum_{i=1}^{N} q_{i}}-c\right) q_{i}-\Gamma_{i}(k)+\frac{\partial V_{i}}{\partial k}\left(G_{i}(k)-q_{i}\right)\right\},
$$


where $V_{i}$ depends on both $k$ and initial time $t$. Differently from the infinite horizon case, we must additionally take into account the transversality conditions on all $V_{i}$ :

$$
\lim _{t \longrightarrow T} V_{i}(k, t)=0 .
$$

Proposition 5. If $G(k)$ is linear, $G(0)=0$ and $\Gamma(k)$ is an $m$-th degree polynomial in $k$, then the system (15) admits the following solution:

$$
V(k, t)=\frac{-\beta_{0}+\frac{a}{N^{2}}}{\rho}\left[1-e^{\rho(t-T)}\right]+\sum_{l=1}^{m} \frac{\beta_{l}}{\alpha l-\rho}\left[1-e^{(\rho-\alpha l)(t-T)}\right] k^{l} .
$$

Proof. As in Proposition 3, call $G(k)=\alpha k$ and $\Gamma(k)=\sum_{l=0}^{m} \beta_{l} k^{l}$. The maximization of the r.h.s. of (15) yields:

$$
\frac{a Q_{-i}}{\left(q_{i}+Q_{-i}\right)^{2}}-c-\frac{\partial V_{i}}{\partial k}=0
$$

then, by assuming symmetry on the relevant variables and functions, i.e. $q_{1}=\ldots=q_{N}=q, V_{1}=\ldots=V_{N}=V, \Gamma_{1}(\cdot)=\ldots=\Gamma_{N}(\cdot)=\Gamma(\cdot), G_{1}(\cdot)=$ $\ldots=G_{N}(\cdot)=G(\cdot)$, we have that (18) yields the following expression for the optimal strategy $q^{*}$ :

$$
q^{*}=\frac{a(N-1)}{N^{2}\left(\frac{\partial V}{\partial k}+c\right)}
$$

which must be plugged into (15) to achieve (the steps are analogous to those in Proposition 3, so we omit them):

$$
-\frac{\partial V(k, t)}{\partial t}+\rho V(k, t)+\sum_{l=0}^{m} \beta_{l} k^{l}-\alpha k \frac{\partial V(k, t)}{\partial k}-\frac{a}{N^{2}}=0 .
$$

We guess a function of the following kind for $V(k, t)$ :

$$
V(k, t)=\sum_{l=0}^{m} A_{l}(t) k^{l}
$$

where $A_{l}(t) \in C^{1}([t, T])$ and the transversality conditions are $A_{l}(T)=0$ for all $l=0,1, \ldots, m$. Plugging (21) into (20), we obtain:

$$
-\sum_{l=0}^{m} \dot{A}_{l}(t) k^{l}+\rho \sum_{l=0}^{m} A_{l}(t) k^{l}-\frac{a}{N^{2}}+\sum_{l=0}^{m} \beta_{l} k^{l}-\alpha k \sum_{l=0}^{m} l A_{l}(t) k^{l-1}=0,
$$


subsequently, all the coefficients of the powers of $k$ are supposed to vanish, giving rise to the following dynamic system:

$$
\left\{\begin{array}{l}
-\dot{A}_{0}(t)+\rho A_{0}(t)-\frac{a}{N^{2}}+\beta_{0}=0 \\
-\dot{A}_{1}(t)+\rho A_{1}(t)-\alpha A_{1}(t)+\beta_{1}=0 \\
\cdots \\
-\dot{A}_{m}(t)+\rho A_{m}(t)-m \alpha A_{m}(t)+\beta_{m}=0
\end{array}\right.
$$

By employing the transversality conditions, we achieve the following unique solutions:

$$
\begin{aligned}
& A_{0}(t)=\frac{-\beta_{0}+\frac{a}{N^{2}}}{\rho}\left[1-e^{\rho(t-T)}\right], \\
& A_{l}(t)=\frac{\beta_{l}}{\alpha l-\rho}\left[1-e^{(\rho-\alpha l)(t-T)}\right],
\end{aligned}
$$

for all $l=1, \ldots, m$. Finally, substituting the found solutions in (21), we obtain the optimal value function in closed form:

$$
V(k, t)=\frac{-\beta_{0}+\frac{a}{N^{2}}}{\rho}\left[1-e^{\rho(t-T)}\right]+\sum_{l=1}^{m} \frac{\beta_{l}}{\alpha l-\rho}\left[1-e^{(\rho-\alpha l)(t-T)}\right] k^{l} .
$$

Corollary 6. If the assumptions of Proposition 5 hold, the optimal feedback strategy is given by:

$$
q^{*}(k, t)=\frac{a(N-1)}{N^{2}\left(\sum_{l=1}^{m} \frac{l \beta_{l}}{\alpha l-\rho}\left[1-e^{(\rho-\alpha l)(t-T)}\right] k^{l-1}+c\right)} .
$$

\section{Applications}

\subsection{The Cournot-Ramsey game}

In this well-known example, in order to produce, firms must accumulate capacity or physical capital $k_{i}(t)$ over time. We chose to consider the kinematic equations for capital accumulation as in Ramsey ([21]), i.e. the following dynamic constraints:

$$
\left\{\begin{array}{l}
\dot{k}_{i}(t)=A k_{i}(t)-q_{i}(t)-\delta k_{i}(t) \\
k_{i}(0)=k_{i 0}>0
\end{array},\right.
$$


where $A k_{i}(t)=y_{i}(t)$ denotes the output produced by firm $i$ at time $t$ and $\delta>0$ denotes the decay rate of capital, equal across firms. I.e., this is the familiar $A k$ version of the Ramsey model. The related cost will be $\Gamma_{i}(t)=b k_{i}(t)$, with $b \geq 0$, representing the aforementioned opportunity cost of a unit of capacity.

Because $G_{i}\left(k_{i}(t)\right)=\alpha k_{i}(t)=(A-\delta) k_{i}(t)$, we can apply all the results collected in the previous Section. In particular, if we posit the following:

$$
\beta_{0}=0, \quad \beta_{1}=b, \quad \beta_{2}=\ldots=\beta_{m}=0, \quad \alpha=A-\delta,
$$

then the application of formulas (13), (14), (22) and (23) respectively entail:

- Infinite horizon:

$$
\begin{gathered}
V^{*}(k)=\frac{b}{A-\delta-\rho} k+\frac{a}{\rho N^{2}}, \\
q^{*}(k)=\frac{a(N-1)(A-\delta-\rho)}{N^{2}[b+c(A-\delta-\rho)]} .
\end{gathered}
$$

- Finite horizon: ${ }^{7}$

$$
\begin{aligned}
V^{*}(k, t) & =\frac{a}{\rho N^{2}}\left[1-e^{\rho(t-T)}\right]+\frac{b\left[1-e^{(\rho-A+\delta)(t-T)}\right]}{A-\delta-\rho} k, \\
q^{*}(k, t) & =\frac{a(N-1)(A-\delta-\rho)}{N^{2}\left[b\left(1-e^{(\rho-A+\delta)(t-T)}\right)+c(A-\delta-\rho)\right]} .
\end{aligned}
$$

In order to ensure the feasibility (i.e., the positivity) of such strategies, we need suitable parametric assumptions:

Proposition 7. In the infinite horizon case, if one of the following conditions:

1. $A>\rho+\delta$,

2. $\delta<A<-\frac{b}{c}+\rho+\delta$,

\footnotetext{
${ }^{7}$ As an ancillary observation, it is worth noting that here, since the feedback optimal strategy coincides with the open-loop one and the strategic contributions cannot be distinguished under symmetry, the co-state variable at initial time appearing in the open-loop formulation of the game, which we omit here for brevity, can be appropriately considered as a shadow price of an additional unit of capacity, while, in general, this is true only of the partial derivative of the value function at initial state (for more on this aspect, see [3]).
} 
holds, then $q^{*}(k)$ is feasible.

Proof. Both conditions are trivially satisfied. In particular, in both cases we have to assume $A>\delta$ to ensure accumulation of capital (otherwise $\dot{k}_{i}(t)$ would be negative at all $t$ ).

Moreover, here $q^{*}(k)$ is constant whereas $k^{*}(t)$ grows unbounded in that $A>\delta$. We can easily compare the optimal output with the one in the Cournot-Nash static setup:

Proposition 8. In the infinite horizon case, we have that:

1. if $A>-\frac{b}{c}+\rho+\delta$, then $q^{C N}>q^{*}(k)$;

2. $A<-\frac{b}{c}+\rho+\delta$, then $q^{*}(k)>q^{C N}$.

Proof. It suffices to evaluate the difference between outputs:

$q^{*}(k)-q^{C N}=\frac{a(N-1)(A-\delta-\rho)}{N^{2}[b+c(A-\delta-\rho)]}-\frac{a(N-1)}{N^{2} c}=-\frac{a b(N-1)(A-\delta-\rho)}{N^{2} c[b+c(A-\delta-\rho)]}$.

In the finite horizon case, the situation is different and we need to establish a time interval over which $q^{*}(k, t)$ is feasible. However, note that at $t=T$ the optimal strategy coincides with the Cournot-Nash optimal strategy: $q(k, T)=q^{C N}$.

Proposition 9. In the finite horizon case we have that:

1. If $\delta<A<-\frac{b}{c}+\rho+\delta, q^{*}(k, t)$ is feasible for each $t \in[0, T)$.

2. If $A>-\frac{b}{c}+\rho+\delta, q^{*}(k, t)$ is feasible for each $t \in(\widetilde{t}, T)$, where

$$
\widetilde{t}=T+\frac{1}{\rho-A+\delta} \ln \left[\frac{c}{b}(A-\rho-\delta)+1\right] .
$$

Proof. We are going to consider the two different cases:

If $A<\rho+\delta$, then the numerator is negative, hence we have to ensure that the denominator is negative too:

$$
b\left(1-e^{(\rho-A+\delta)(t-T)}\right)+c(A-\delta-\rho)<0 \Longleftrightarrow \ldots \Longleftrightarrow
$$




$$
\Longleftrightarrow e^{(\rho-A+\delta)(t-T)}>\frac{c}{b}(A-\delta-\rho)+1
$$

If the r.h.s. is negative, i.e. $A<-\frac{b}{c}+\rho+\delta, q^{*}(k, t)$ is positive for all $t \in[0, T)$. If the r.h.s. is positive, then:

$$
t-T>\frac{1}{\rho-A+\delta} \ln \left[\frac{c}{b}(A-\rho-\delta)+1\right]
$$

hence if we call $\tilde{t}=T+\frac{1}{\rho-A+\delta} \ln \left[\frac{c}{b}(A-\rho-\delta)+1\right], q^{*}(k, t)>0$ over $(\widetilde{t}, T)$.

Subsequently, consider $A>\rho+\delta$, we have to prove the positivity of the denominator of $q^{*}(k, t)$ :

$$
\begin{gathered}
b\left(1-e^{(\rho-A+\delta)(t-T)}\right)+c(A-\delta-\rho)>0 \Longleftrightarrow \ldots \Longleftrightarrow \\
\Longleftrightarrow t-T>\frac{1}{\rho-A+\delta} \ln \left[\frac{c}{b}(A-\rho-\delta)+1\right],
\end{gathered}
$$

then $q^{*}(k, t)>0$ over $(\widetilde{t}, T)$, meaning that the relevant condition for the restriction of the time interval is $A>-\frac{b}{c}+\rho+\delta$.

The next Figures sketch the behaviour of the optimal strategy in finite horizon, showing the difference between its possible domains $(\widetilde{t}, T]$ and $[0, T]$ in compliance with Proposition 9.

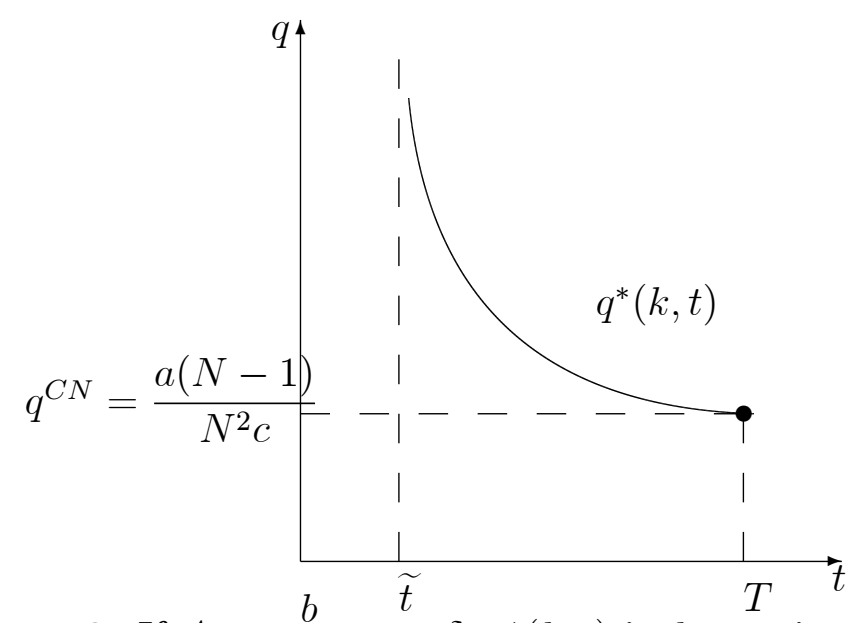

Figure 2. If $A>-\frac{b}{c}+\stackrel{t}{t}+\delta, q^{*}(k, t)$ is decreasing and it reaches $q^{C N}$ at the instant $T$. 


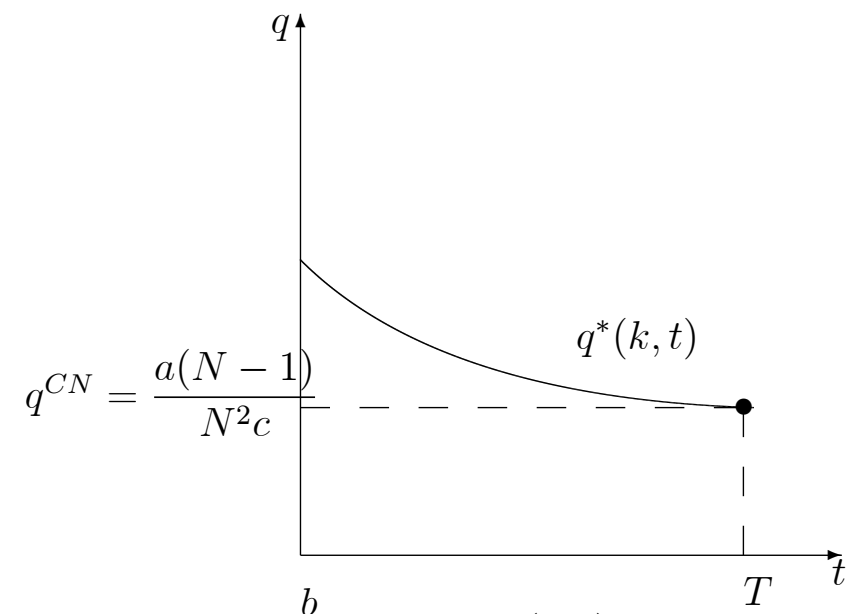

Figure 3. If $A>-\frac{b}{c}+\rho+\delta, q^{*}(k, t)$ is decreasing and it reaches $q^{C N}$ at the instant $T$.

Corollary 10. Since the differential game at hand is a linear state one ${ }^{8}$, the open-loop equilibrium is subgame perfect as it coincides with the feedback equilibrium $q^{*}(k, t)$ yielded by the Bellman equation. Moreover, this would hold true also in the more general case where $y_{i}(t)=f\left(k_{i}(t)\right)$, with $f^{\prime}\left(k_{i}(t)\right)>0$ and $f^{\prime \prime}\left(k_{i}(t)\right) \leq 0$. That is, state-linearity is not necessary to yield subgame perfection in a Cournot-Ramsey game. For more on this issue, see [4] and [6].

If we substitute $q^{*}(k, t)$ in the dynamic constraint $(24)$, we can also achieve the expression of the optimal state $k^{*}(t)$ :

$$
\left\{\begin{array}{l}
\dot{k}_{i}(t)=(A-\delta) k_{i}(t)-\frac{a(N-1)(A-\delta-\rho)}{N^{2}\left[b\left(1-e^{(\rho-A+\delta)(t-T)}\right)+c(A-\delta-\rho)\right]}, \\
k_{i}(0)=k_{i 0}
\end{array},\right.
$$

whose unique solution is given by:

$k^{*}(t)=\left(k_{0}-\frac{a(N-1)(A-\delta-\rho)}{N^{2}} \int_{0}^{t} \frac{e^{-(A-\delta) s}}{b\left(1-e^{(\rho-A+\delta)(s-T)}\right)+c(A-\delta-\rho)} d s\right) e^{(A-\delta) t}$.

Now the joint feasibility of $q^{*}(t)$ and $k^{*}(t)$ can be evaluated:

\footnotetext{
${ }^{8} \mathrm{~A}$ linear state game is one where, if we call $\mathcal{H}_{i}(\cdot)$ the $i$-th firm's Hamiltonian function, we have that: $\frac{\partial^{2} \mathcal{H}_{i}(\cdot)}{\partial q_{i} \partial k_{j}}=\frac{\partial^{2} \mathcal{H}_{i}(\cdot)}{\partial k_{j}^{2}}=0$ for all $i, j=1, \ldots, N$. For more on linear state games, see [10](ch. 7), inter alia.
} 
Proposition 11. Under the same assumptions as in Proposition 9, if $q^{*}(t)$ is feasible and if

$$
k_{0}>\frac{a(N-1)(A-\delta-\rho)}{N^{2}} \int_{0}^{t} \frac{e^{-(A-\delta) s}}{b\left(1-e^{(\rho-A+\delta)(s-T)}\right)+c(A-\delta-\rho)} d s
$$

for all $t \in(0, T]$, then $k^{*}(t)$ is feasible as well.

Proof. It immediately follows from the positivity of $q^{*}(t)$ and from the expression (25).

The next Proposition provides the exact expression of $k^{*}(t)$ :

Proposition 12. The optimal state of the Cournot-Ramsey game is given by the following function:

$$
\begin{gathered}
k^{*}(t)=\left[k_{0}-\frac{a(N-1)(A-\delta-\rho)}{N^{2}}\left(\frac{e^{-(A-\delta-\rho) T}}{\rho}\left(e^{-\rho t}-1\right)+\right.\right. \\
\left.\left.+\frac{b+c(A-\delta-\rho)}{\rho e^{-2(A-\delta-\rho) T}}\left(\log \left(\frac{e^{-(A-\delta-\rho) T}-(b+c(A-\delta-\rho)) e^{-(A-\delta-\rho) t}}{e^{-(A-\delta-\rho) T}-(b+c(A-\delta-\rho))}\right)\right)\right)\right] e^{(A-\delta) t} .
\end{gathered}
$$

Proof. The explicit calculation of (25) needs the calculation of the related integral:

$$
I(t)=\int_{0}^{t} \frac{e^{-(A-\delta) s}}{C_{1}-C_{2} e^{(\rho-A+\delta) s}} d s,
$$

where $C_{1}=b+c(A-\delta-\rho)$ and $C_{2}=e^{-(\rho-A-\delta) T}$. We have that:

$$
\begin{gathered}
I(t)=\int_{0}^{t} \frac{\left(-C_{2}\right) e^{-(A-\delta-\rho) s}+C_{1}-C_{1}}{\left(-C_{2}\right) e^{\rho s}\left(C_{1}-C_{2} e^{(\rho-A+\delta) s}\right)} d s=\frac{1}{C_{2}}\left[\frac{e^{-\rho s}}{\rho}\right]_{0}^{t}+\frac{C_{1}}{C_{2}} \int_{0}^{t} \frac{1}{e^{\rho s}\left(C_{1}-C_{2} e^{(\rho-A-\delta) s}\right)} d s= \\
=\frac{1}{\rho C_{2}}\left[e^{-\rho t}-1\right]+\frac{C_{1}}{C_{2}} \int_{0}^{t} \frac{1}{e^{\rho s}\left(C_{1}-C_{2} e^{(\rho-A-\delta) s}\right)} d s .
\end{gathered}
$$

Then, applying the change of variable $x=e^{-\rho s}$, leading to the change of differential $d s=-\frac{d x}{\rho x}$, we obtain:

$$
\begin{aligned}
I(t) & =\frac{1}{\rho C_{2}}\left[e^{-\rho t}-1\right]-\frac{C_{1}}{\rho C_{2}} \int_{1}^{e^{-\rho t}} \frac{d x}{C_{1}-C_{2} x^{\frac{A-\delta-\rho}{\rho}}} d s= \\
& =\frac{1}{\rho C_{2}}\left[e^{-\rho t}-1\right]+\frac{1}{\rho C_{2}} \int_{e^{-\rho t}}^{1} \frac{d x}{1-\frac{C_{2}}{C_{1}} x^{\frac{A-\delta-\rho}{\rho}}} d s=
\end{aligned}
$$




$$
\begin{gathered}
=\frac{1}{\rho C_{2}}\left[e^{-\rho t}-1\right]+\frac{1}{\rho C_{2}} \int_{e^{-\rho t}}^{1} \sum_{k=0}^{\infty}\left(\frac{C_{2}}{C_{1}} x^{\frac{A-\delta-\rho}{\rho}}\right)^{k} d s= \\
=\frac{1}{\rho C_{2}}\left[e^{-\rho t}-1\right]+\frac{1}{\rho C_{2}}\left[\sum_{k=0}^{\infty} \frac{\left(\frac{C_{2}}{C_{1}}\right)^{k}\left(x^{\frac{A-\delta-\rho}{\rho}}\right)^{k+1}}{k+1}\right]_{e^{-\rho t}}^{1}= \\
=\frac{1}{\rho C_{2}}\left[e^{-\rho t}-1\right]+\frac{1}{\rho C_{2}}\left[\frac{C_{1}}{C_{2}} \sum_{l=1}^{\infty} \frac{\left(\frac{C_{2}}{C_{1}}\right)^{l}}{l}-\frac{C_{1}}{C_{2}} \sum_{l=1}^{\infty} \frac{\left.\left(\frac{C_{2}}{C_{1}} e^{-(A-\delta-\rho) t}\right)^{l}\right]}{l}\right]= \\
=\frac{1}{\rho C_{2}}\left[e^{-\rho t}-1\right]+\frac{C_{1}}{\rho C_{2}^{2}}\left[-\log \left(1-\frac{C_{1}}{C_{2}}\right)+\log \left(1-\frac{C_{1}}{C_{2}} e^{-(A-\delta-\rho) t}\right)\right]= \\
=\frac{1}{\rho C_{2}}\left[e^{-\rho t}-1\right]+\frac{C_{1}}{\rho C_{2}^{2}}\left[\log \left(\frac{C_{2}-C_{1} e^{-(A-\delta-\rho) t}}{C_{2}-C_{1}}\right)\right] .
\end{gathered}
$$

Finally, plugging (27) into (25) yields the complete expression (26)of the optimal capital:

$$
\begin{gathered}
k^{*}(t)=\left[k_{0}-\frac{a(N-1)(A-\delta-\rho)}{N^{2}} I(t)\right] e^{(A-\delta) t}= \\
=\left[k_{0}-\frac{a(N-1)(A-\delta-\rho)}{N^{2}}\left(\frac{e^{-(A-\delta-\rho) T}}{\rho}\left(e^{-\rho t}-1\right)+\right.\right. \\
\left.\left.+\frac{b+c(A-\delta-\rho)}{\rho e^{-2(A-\delta-\rho) T}}\left(\log \left(\frac{e^{-(A-\delta-\rho) T}-(b+c(A-\delta-\rho)) e^{-(A-\delta-\rho) t}}{e^{-(A-\delta-\rho) T}-(b+c(A-\delta-\rho))}\right)\right)\right)\right] e^{(A-\delta) t} .
\end{gathered}
$$

Given (26), we can evaluate $k^{*}(T)$, i.e. the terminal value of capital at the end of the time interval ${ }^{9}$ :

$$
\begin{aligned}
& k^{*}(T)=\left[k_{0}-\frac{a(N-1)(A-\delta-\rho)}{N^{2}} I(T)\right] e^{(A-\delta) T}=-\frac{a(N-1)(A-\delta-\rho)}{\rho N^{2}}\left(1-e^{\rho T}+\right. \\
& \left.+\frac{b+c(A-\delta-\rho)}{e^{-(3(A-\delta)-2 \rho) T}}\left(\log \left(\frac{1-[b+c(A-\delta-\rho)]}{1-[b+c(A-\delta-\rho)] e^{(A-\delta-\rho) T}}\right)\right)\right)+k_{0} e^{(A-\delta) T} .
\end{aligned}
$$

\footnotetext{
${ }^{9}$ We omit the most tedious calculations, reminding the readers that all of them are available upon request to the authors.
} 
If we call $\pi^{*}=\pi\left(q^{*}(T), k^{*}(T)\right)$ the profit function evaluated at the terminal instant $T$, we are able to compare it with the profit function $\pi^{C N}=\pi\left(Q^{C N}\right)$ evaluated at the steady state in the static Cournot problem as shown in Subsection 2.1. Namely, we have that:

$$
\begin{gathered}
\pi^{*}=\left(\frac{a}{N q_{i}^{*}(T)}-c\right) q_{i}^{*}(T)-b k_{i}^{*}(T)=\frac{a}{N}-c q^{*}(T)-b k^{*}(T)= \\
=\frac{a}{N^{2}}-b\left[k_{0} e^{(A-\delta) T}-\frac{a(N-1)(A-\delta-\rho)}{N^{2}}\left(1-e^{\rho T}\right)+\right. \\
\left.+\frac{b+c(A-\delta-\rho)}{\rho e^{-(3(A-\delta)-2 \rho) T}}\left(\log \left(\frac{1-[b+c(A-\delta-\rho)]}{1-[b+c(A-\delta-\rho)] e^{(A-\delta-\rho) T}}\right)\right)\right] .
\end{gathered}
$$

Proposition 13. Under the hypotheses of Propositions 9 and 11, if $q^{*}(t)$ and $k^{*}(t)$ are both feasible at all $t \in[0, T]$, then the Cournot-Nash equilibrium profit is larger than the Ramsey-Cournot equilibrium level at the terminal instant.

Proof. It suffices to consider the difference:

$$
\pi^{*}-\pi^{C N}=\frac{a}{N^{2}}-b k^{*}(T)-\frac{a}{N^{2}}=-b k^{*}(T)
$$

which is strictly negative by the feasibility of $k^{*}(t)$ at all instants, meaning that the Cournot-Nash equilibrium profit exceeds the Ramsey-Cournot terminal profit.

Remark 14. It is worth noting that comparing the two optimal strategies in the static and in the dynamic cases, one immediately sees that the presence of capital accumulation in the dynamic game plays a key role in opening the way towards a solution to the indeterminacy issue affecting the static game as the marginal production cost c of the consumption good drops to zero. Essentially, if $c=0$, no solution exists for the static game if no strategy space is compact, whereas in the differential game with capacity accumulation $q^{*}(k, t)$ is well-defined and feasible under suitable parametric conditions even when the marginal cost is zero, both over finite and infinite horizons.

Propositions 8 and 13 suggest that at the subgame perfect equilibrium of the dynamic game the representative firm may produce either more or less but she earns higher profits when she plays the Cournot-Nash equilibrium of the static game, irrespective of the levels of marginal cost, opportunity cost, intensity of capacity accumulation growth and intertemporal discount rate. 
Having characterised the subgame perfect equilibrium of the differential game, we can now proceed to the analysis of its application to horizontal mergers.

\subsection{Horizontal mergers}

To illustrate the advantages of our approach to the feedback solution of the differential oligopoly game à la Ramsey, we illustrate here its applicability to the analysis of the private profitability of a horizontal merger, and its welfare appraisal.

As is well known, a lively debate has taken place on this topic from the 1980's, based upon static oligopoly models. A thorough overview of it is outside the scope of the present paper, and it will suffice to recollect a few essential aspects. Examining a Cournot industry with constant returns to scale, in [22] it is shown that a large proportion of the population of firms has to participate in the merger in order for the latter to be profitable. In particular, a striking result of their analysis is that, in the triopoly case, bilateral mergers are never profitable. Enriching the picture by allowing for the presence of convex variable costs and fixed costs, one may find a way out of this puzzle (in particular, see [20] and [12]).

Now take the static Cournot game and examine the incentive for $M>1$ firms to merge horizontally, out of the initial $N$. After the merger (if it does take place), there remain $N-M+1$ firms. If we call $\pi^{C N}(j)$ the profit of a firm in the Cournot static game among $j$ firms, without distinguishing the original ones from those generated by the merger, we can prove the following:

Proposition 15. In the static Cournot game with hyperbolic inverse demand, the merger is profitable if and only if $M-\sqrt{M}<N<M+\sqrt{M}$.

Proof. Profitability holds when

$$
\begin{gathered}
\frac{\pi^{C N}(N-M+1)}{M}>\pi^{C N}(N) \Longleftrightarrow \frac{a}{M(N-M+1)^{2}}>\frac{a}{N^{2}} \Longleftrightarrow \\
\Longleftrightarrow N^{2}>M(N-M+1)^{2} \Longleftrightarrow \ldots \Longleftrightarrow N^{2}-2 M N-M(1-M)<0,
\end{gathered}
$$

which entails $N \in(M-\sqrt{M}, M+\sqrt{M})$.

It is easily checked that, contrary to [22], if $N=3$ and $M=2$, the merger is profitable. 
On the other hand, if we consider the terminal outcome of the differential game over finite horizon, in compliance with the above notation, we can assess the profit incentive scheme for an $M$-firm merger in the dynamic framework too.

If we consider the assumptions of Proposition 9 to ensure the feasibility of the optimal strategy on $[0, T]$, we can state the following:

Proposition 16. If a horizontal merger of $M$ firms is profitable in the Cournot static game, then if $\delta<A<-\frac{b}{c}+\delta+\rho$, the same merger is profitable in the Cournot-Ramsey game on the horizon $[0, T]$ as well.

Proof. The profitability of a merger in the Cournot-Ramsey game is measured by

$$
\frac{\pi^{*}(N-M+1)-b k_{N-M+1}^{*}(T)}{M}>\pi^{*}(N)-b k_{N}^{*}(T),
$$

where we called $k_{l}^{*}(T)$ the capital at time $T$ under circumstances where a merger of $l$ firms took place. In order to simplify the notation, call

$g(T)=\left(1-e^{\rho T}+\frac{b+c(A-\delta-\rho)}{e^{-(3(A-\delta)-2 \rho) T}}\left(\log \left(\frac{1-[b+c(A-\delta-\rho)]}{1-[b+c(A-\delta-\rho)] e^{(A-\delta-\rho) T}}\right)\right)\right)$

as in (28). The inequality (30) becomes:

$$
\begin{gathered}
\frac{a}{M(N-M+1)^{2}}-\frac{b}{M}\left[-\frac{a(N-M)(A-\delta-\rho)}{(N-M+1)^{2}} g(T)+k_{0} e^{(A-\delta) T}\right]> \\
>\frac{a}{N^{2}}-b\left[-\frac{a(N-1)(A-\delta-\rho)}{N^{2}} g(T)+k_{0} e^{(A-\delta) T}\right],
\end{gathered}
$$

which amounts to

$$
\begin{aligned}
& a\left[\frac{N^{2}-M(N-M+1)^{2}}{M N^{2}(N-M+1)^{2}}\right]-b k_{0} e^{(A-\delta) T}\left(\frac{1}{M}-1\right)+ \\
& +b a(A-\delta-\rho) g(T)\left[\frac{N-M}{M(N-M+1)^{2}}-\frac{N-1}{N^{2}}\right]>0 .
\end{aligned}
$$

By Proposition 15, the first term of (31) is positive when the $M$-firm merger is profitable in the static framework, the second term is positive for all $M>1$, whereas the remaining term is positive for $A<-\frac{b}{c}+\delta+\rho<\delta+\rho$ (the condition ensuring feasibility of the strategy over $[0, \stackrel{c}{T}]$ by Proposition 
$9)$ if and only if the quantity $\frac{N-M}{M(N-M+1)^{2}}-\frac{N-1}{N^{2}}$ is negative. In fact, we have that:

$$
\begin{gathered}
\frac{N-M}{M(N-M+1)^{2}}-\frac{N-1}{N^{2}}=\frac{N-M}{M(N-M+1)^{2}}-\frac{1}{N}+\frac{1}{N^{2}}< \\
<\frac{N-M}{M(N-M+1)^{2}}-\frac{1}{N}+\frac{1}{M(N-M+1)^{2}}=\frac{1}{M(N-M+1)}-\frac{1}{N}= \\
=\frac{N-M N+M^{2}-M}{N M(N-M+1)}=\frac{(N-M)(1-M)}{N M(N-M+1)}<0,
\end{gathered}
$$

where we exploited the inequality $N^{2}>M(N-M+1)^{2}$. Hence, this completes the proof.

Taken together, these facts entail that the interval wherein the $M$-firm merger is profitable may be the same in the dynamic setup and in the static one, given that the measure of output productivity $A$ is small enough.

The examination of the welfare consequences of a merger is omitted, as it goes without saying that any merger would diminish social welfare, both in the static as well as in the dynamic setting. This is trivially due to the fact that the damage caused to consumer surplus always outweighs the increase in industry profits. ${ }^{10}$

\section{Concluding remarks}

We characterised the subgame perfect equilibrium of a dynamic Cournot game with inverse hyperbolic demand and costly capacity accumulation, showing that the feedback solution, coincident with the open-loop one, is subgame perfect. We fully carried out the calculation of the optimal value functions and of the strategies of the differential game subject to a Ramsey-type dynamic constraint. Then, we employed the model to analyse the feasibility of horizontal mergers in both static and dynamic settings, finding out appropriate parametric conditions under which the profitability of a merger in a static game implies the profitability in a dynamic game as well.

\footnotetext{
${ }^{10}$ In line of principle, a merger could allow for some reduction in the total opportunity costs for the industry, giving rise to a possible efficiency defense argument (see [12]). Although we omit the related calculations for brevity, it is quickly checked that this never outweighs the loss in consumer surplus necessarily generated by any merger. Hence, in this model the efficiency argument cannot be advocated to justify the merger itself.
} 
Possible future developments of our findings consist in the analysis of the feedback information structure of further differential oligopoly games endowed with a hyperbolic inverse demand function, possibly in presence of more complex dynamic constraints and in asymmetric frameworks. 


\section{References}

[1] Anderson, S.P. and M. Engers (1992), "Stackelberg vs Cournot Oligopoly Equilibrium", International Journal of Industrial Organization, 10, 127-135.

[2] Benchekroun, H. (2003), "The Closed-Loop Effect and the Profitability of Horizontal Mergers", Canadian Journal of Economics, 36, 546-565.

[3] Caputo, M.R. (2007), "The Envelope Theorem for Locally Differentiable Nash Equilibria of Finite Horizon Differential Games", Games and Economic Behavior, 61, 198-224.

[4] Cellini, R., and L. Lambertini (1998), "A Dynamic Model of Differentiated Oligopoly with Capital Accumulation", Journal of Economic Theory, 83, 145-155.

[5] Cellini, R. and L. Lambertini (2007), "Capital Accumulation, Mergers, and the Ramsey Golden Rule", in M. Quincampoix, T. Vincent and S. Jørgensen (eds), Advances in Dynamic Game Theory and Applications, Annals of the International Society of Dynamic Games, vol. 8, Boston, Birkhäuser, 487-505.

[6] Cellini, R. and L. Lambertini (2008), "Weak and Strong Time Consistency in a Differential Oligopoly Game with Capital Accumulation", Journal of Optimization Theory and Applications, 138, 17-126.

[7] Deaton, A. and J. Muellbauer (1980), Economics and Consumer Behavior, Cambridge, Cambridge University Press.

[8] Dixit, A.K. (1986), "Comparative Statics for Oligopoly", International Economic Review, 27, 107-122.

[9] Dockner, E., and A. Gaunersdorfer (2001), "On the Profitability of Horizontal Mergers in Industries with Dynamic Competition", Japan and the World Economy, 13, 195-216.

[10] Dockner, E.J., S. Jørgensen, N.V. Long and G. Sorger (2000), Differential Games in Economics and Management Science, Cambridge, Cambridge University Press.

[11] Esfahani, H. and L. Lambertini (2012), "The profitability of small horizontal mergers with nonlinear demand functions", Operations Research Letters, 40, 370-373. 
[12] Farrell, J. and C. Shapiro (1990), "Horizontal Mergers: An Equilibrium Analysis", American Economic Review, 80, 107-126.

[13] Fershtman, C. and M.I. Kamien (1987), "Dynamic Duopolistic Competition with Sticky Prices", Econometrica, 55, 1151-1164.

[14] Friedman, J.W. (1977), Oligopoly and the Theory of Games, Amsterdam, North-Holland.

[15] Hausman, J.A. (1981), "Exact Consumer's Surplus and Deadweight Loss", American Economic Review, 71, 662-676.

[16] Jun, B. and X. Vives (2004), "Strategic Incentives in Dynamic Duopoly", Journal of Economic Theory, 116, 249-281.

[17] Koulovatianos, C. and L. Mirman (2007), "The Effects of Market Structure on Industry Growth: Rivalrous Non-Excludible Capital", Journal of Economic Theory, 133, 199-218.

[18] Lambertini, L. (2010), "Oligopoly with Hyperbolic Demand: A Differential Game Approach", Journal of Optimization Theory and Applications, 145, 108-119.

[19] Long, N.V. (2010), A Survey of Dynamic Games in Economics, Singapore, World Scientific.

[20] Perry, M.K. and R.H. Porter (1985), "Oligopoly and the Incentive for Horizontal Merger", American Economic Review, 75, 219-227.

[21] Ramsey, F.P. (1928), "A Mathematical Theory of Saving", Economic Journal, 38, 543-549. Reprinted in Stiglitz, J.E. and H. Uzawa (1969, eds.), Readings in the Modern Theory of Economic Growth, Cambridge, MA, MIT Press.

[22] Salant, S.W., S. Switzer and R.J. Reynolds (1983), "Losses from Horizontal Merger: The Effects of an Exogenous Change in Industry Structure on Cournot-Nash Equilibrium", Quarterly Journal of Economics, 98, 185-213.

[23] Shy, O. (1995), Industrial Organization. Theory and Applications, Cambridge, MA, MIT Press.

[24] Simaan, M. and T. Takayama (1978), "Game Theory Applied to Dynamic Duopoly Problems with Production Constraints", Automatica, 14, 161-166. 
[25] Varian, H.R. (1982), "The Nonparametric Approach to Demand Analysis", Econometrica, 50, 945-973.

[26] Varian, H.R. (1990), "Goodness of Fit in Optimizing Models", Journal of Econometrics, 46, 125-140.

[27] Varian, H.R. (1992), Microeconomic Analysis. Third Edition, New York, Norton. 


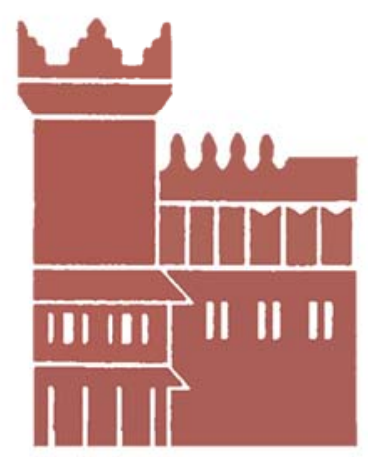

Alma Mater Studiorum - Università di Bologna DEPARTMENT OF ECONOMICS

Strada Maggiore 45

40125 Bologna - Italy

Tel. +39051 2092604

Fax +390512092664

http://www.dse.unibo.it 